\title{
Clinicopathological significance of somatic RNF43 mutation and aberrant expression of ring finger protein 43 in intraductal papillary mucinous neoplasms of the pancreas
}

Hitomi Sakamoto ${ }^{1,2}$, Yuko Kuboki ${ }^{1}$, Takashi Hatori ${ }^{3}$, Masakazu Yamamoto ${ }^{3}$, Masanori Sugiyama ${ }^{2}$, Noriyuki Shibata ${ }^{4}, K_{\text {Kyoko Shimizu }}^{5}$, Keiko Shiratori ${ }^{5}$ and Toru Furukawa ${ }^{1}$

${ }^{1}$ Institute for Integrated Medical Sciences, Tokyo Women's Medical University, Tokyo, Japan; ${ }^{2}$ Department of Surgery, Kyorin University School of Medicine, Mitaka, Japan; ${ }^{3}$ Department of Gastroenterological Surgery, Tokyo Women's Medical University, Tokyo, Japan; ${ }^{4}$ Department of Pathology, Tokyo Women's Medical University, Tokyo, Japan and ${ }^{5}$ Department of Gastroenterology, Tokyo Women's Medical University, Tokyo, Japan

Mutations in RNF43, which encodes the ubiquitin E3 ligase ring finger protein 43, were recently found in intraductal papillary mucinous neoplasms of the pancreas. We evaluated somatic mutations of RNF43 and the expression of ring finger protein $\mathbf{4 3}$ as well as their associations with the molecular and clinicopathological features in 176 surgically resected intraductal papillary mucinous neoplasms. Frozen tissues were available for 57 cases and were used for next-generation sequencing analysis of the entire coding exons of RNF43. Formalin-fixed and paraffinembedded tissues from all 176 cases were used for the immunohistochemical analysis of the expression of ring finger protein 43. Mutations detected with the next-generation sequencing analysis were validated by using Sanger sequencing. Statistical analysis was used to evaluate the associations between RNF43 aberrations and molecular and clinicopathological features including GNAS mutations, KRAS mutations, loss of SMA and MAD4 homologue expression, tumor protein 53 overexpression, tumor grade, histological type, mural nodule detection, macroscopic type, stage, recurrence, and survival. Somatic RNF43 mutations were found in $8(14 \%)$ of the 57 examined cases, and included 5 frameshift mutations (p.F69fs, p.S264fs, p.L311fs, p.R363fs, and p.V490fs), 1 non-sense mutation (p.Q153X), and 2 missense mutations (p.I164N and p.P310A). The expression of ring finger protein 43 was downregulated in $52(29.5 \%)$ of the 176 examined cases. RNF43 mutations were significantly associated with the downregulated expression of ring finger protein $43(P=0.011)$, GNAS mutation $(P=0.020)$, and mural nodule detection $(P=0.038)$. The expression of ring finger protein 43 was not associated with any clinicopathological features except RNF43 mutation. These results indicate that RNF43 mutation might cause downregulation of the expression of ring finger protein $\mathbf{4 3}$ and play a crucial role and associate synergistically with GNAS mutation during development of intraductal papillary mucinous neoplasm of the pancreas.

Modern Pathology (2015) 28, 261-267; doi:10.1038/modpathol.2014.98; published online 1 August 2014

Intraductal papillary mucinous neoplasm of the pancreas is a primarily non-invasive tumor characterized by a dilated, mucin-filled duct. ${ }^{1}$ The dilated

Correspondence: Professor T Furukawa, MD, PhD, Institute for Integrated Medical Sciences, Tokyo Women's Medical University, 8-1 Kawadacho, Shinjuku, Tokyo 162-8666, Japan.

E-mail: furukawa.toru@twmu.ac.jp

Received 4 April 2014; accepted 22 May 2014; published online 1 August 2014 duct is lined with neoplastic epithelial cells exhibiting a papillary growth pattern with varying degrees of atypia and abundant mucin secretion; this appearance differs greatly from that of a conventional pancreatic cancer such as ductal adenocarcinoma that forms a highly invasive, firm mass. ${ }^{2}$ However, intraductal papillary mucinous neoplasms often accompany or progress to ductal adenocarcinoma, resulting in a poor prognosis; therefore, intraductal papillary mucinous neoplasm 
is regarded as a precursor of ductal adenocarcinoma. ${ }^{1}$ Currently, given the lack of appropriate biomarkers to monitor the disease phenotype, diagnosis and treatment of intraductal papillary mucinous neoplasms rely heavily on imaging studies in accordance with a guideline for the management of patients with these neoplasms, ${ }^{3}$ thus indicating a pressing need to develop molecular markers on the basis of the molecular phenotypes. In 2011, it was discovered that intraductal papillary mucinous neoplasms often harbored mutations in GNAS and RNF43.4-6 Most GNAS mutations found in intraductal papillary mucinous neoplasms are either R201H or R201C, which are known to induce gain of function of the encoded protein guanosine nucleotide-binding protein alpha. ${ }^{7}$ In contrast, RNF43 mutations are mostly frameshift or nonsense mutations that are expected to induce a lossof-function of the encoded protein ring finger protein 43. ${ }^{4,5}$ Among pancreatic neoplasms, GNAS and RNF43 mutations are almost exclusively found in intraductal papillary mucinous neoplasms, and therefore such mutations are thought to play crucial roles in the characteristic phenotypes of intraductal papillary mucinous neoplasms. ${ }^{4,5}$

Ring finger protein 43 is a ubiquitin E3 ligase that targets the frizzled receptor via interactions with the R-spondin protein. ${ }^{8}$ Ubiquitination of the frizzled receptor results in the blocking of signal transduction through the wingless-type MMTV integration site (Wnt) pathway; accordingly, loss of function of ring finger protein 43 leads to Wnt signaling activation and tumorigenesis in the colon. ${ }^{8}$ RNF43 mutations have also been shown to confer Wnt dependency in pancreatic cancer cells. ${ }^{9}$ However, the clinicopathological relevance of RNF43 mutations in intraductal papillary mucinous neoplasms remains unclear.

Herein, we evaluated RNF43 mutations and expression as well as the associations with the molecular and clinicopathological features of intraductal papillary mucinous neoplasms.

\section{Materials and methods}

\section{Tissues}

A total of 176 patients with intraductal papillary mucinous neoplasms who were surgically treated between 2001 and 2010 at the Tokyo Women's Medical University Hospital were evaluated. Formalin-fixed and paraffin-embedded tissues from the 176 patients were used for the immunohistochemical examination. Frozen tissues from 57 of the 176 patients were used for next-generation sequencing analysis. The clinicopathological features of the included cases are listed in Table 1. This study was approved by the Ethics Committee of the Tokyo Women's Medical University.
Table 1 Clinicopathological features of studied cases

\begin{tabular}{|c|c|}
\hline Total & 176 \\
\hline \multicolumn{2}{|l|}{ GNAS } \\
\hline Mutant & 80 \\
\hline Wild & 96 \\
\hline \multicolumn{2}{|l|}{ KRAS } \\
\hline Mutant & 96 \\
\hline Wild & 80 \\
\hline \multicolumn{2}{|l|}{ SMAD4 expression } \\
\hline Retain & 154 \\
\hline Loss & 22 \\
\hline \multicolumn{2}{|l|}{ TP53 expression } \\
\hline Overexpression & 25 \\
\hline Normal & 151 \\
\hline \multicolumn{2}{|l|}{ Grade and invasion } \\
\hline Low grade & 91 \\
\hline High grade & 37 \\
\hline Invasive & 48 \\
\hline \multicolumn{2}{|l|}{ Туре } \\
\hline Gastric & 101 \\
\hline Intestinal & 56 \\
\hline Oncocytic & 8 \\
\hline Pancreatobiliary & 11 \\
\hline \multicolumn{2}{|l|}{ Mural nodule } \\
\hline Detected & 129 \\
\hline Not detected & 47 \\
\hline \multicolumn{2}{|l|}{ Macroscopic type } \\
\hline Branch & 83 \\
\hline Main & 51 \\
\hline Mixed & 42 \\
\hline \multicolumn{2}{|l|}{ Stage $^{\mathrm{a}}$} \\
\hline $0 \mathrm{a}$ & 81 \\
\hline 0 & 35 \\
\hline 1 & 8 \\
\hline 2 & 10 \\
\hline 3 & 19 \\
\hline $4 a$ & 8 \\
\hline $4 b$ & 3 \\
\hline \multicolumn{2}{|l|}{ Recurrence } \\
\hline Recurred & 31 \\
\hline Not recurred & 145 \\
\hline \multicolumn{2}{|l|}{ Prognosis } \\
\hline 5-year survival rate & 0.904 \\
\hline
\end{tabular}

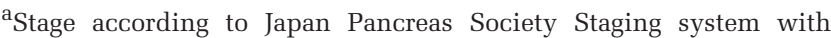
modification with Stage 0a indicating low-grade tumor (Japan Pancreas Society. Classification of Pancreatic Carcinoma, 3rd edn. Kanehara \& Co. Ltd.: Tokyo, 2011).

\section{Next-Generation Sequencing}

Methanol-fixed and hematoxylin-stained frozen sections were prepared from frozen tissues. Tumor tissues were collected from each section by manual dissection under microscopic guidance. In noninvasive cases, a representative intraductal lesion in each case showing the highest grade of atypia was 
Table 2 Primers used for validation of RNF43 mutations

\begin{tabular}{llllll}
\hline GRCh37/hg19 & Exon & Label & Forward & Reverse & Annealing $\left({ }^{\circ} \mathrm{C}\right)$ \\
\hline Ch17:56435671 & Exon 9 & G25-26 & CCAGCCAGTGACTCCAGCTC & GCTGGGGATCCCCTTTAGGG \\
Ch17:56436050 & Exon 9 & G27-28 & ACCAGGTCGAAGACTCCACC & TGATGCCGAGGGCCCATGCC & 62 \\
Ch17:56437534 & Exon 8 & G29-30 & GCTACGGGTCATTTCCTGCC & TGTCTGCCTACACAGAGGGG & 62 \\
Ch17:56438207 & Exon 7 & G17-18 & CCGCTTCAGCAGAGAACAGC & TGGTCATGGAGGTGAACCAC \\
Ch17:56440727 & Exon 5 & G31-32 & AAGTCACAGCAGCCCTGTG & GCTCAATCCTCACATGGGCC & 62 \\
Ch17:56440761 & Exon 5 & G19-20 & AGATAAAGCTCTCAGGGGAG & GGTTCTTGTACACAAACTCC & 62 \\
Ch17:56492734 & Exon 2 & G21-22 & GGAGTCTGAAAGATCAGCAG & CATATTTCAAACAGATGGAAGTG & 55 \\
Ch17:56492734 & Exon 2 & G13-14 & TTATCAGAGTGATCCCCTTG & CTTGCCTGCATTAATTTCC & 58 \\
\hline
\end{tabular}

aNested primers.

dissected and collected. In invasive cases, ie, cases with intraductal papillary mucinous neoplasm with an associated invasive carcinoma, an area containing invasive carcinoma was dissected and collected. Genomic DNA was extracted from the collected tissues by using the Charge Switch ${ }^{\circledR}$ gDNA Micro Tissue kit (Life Technologies, Carlsbad, CA, USA). The extracted DNA was used for semiconductor sequencing analysis with an Ion AmpliSeq ${ }^{\mathrm{TM}}$ Primer Pool custom designed for all RNF43 coding regions, Ion AmpliSeq ${ }^{\mathrm{TM}}$ Library Kit, Ion Xpress ${ }^{\mathrm{TM}}$ Library Barcode Adaptors, Ion One Touch ${ }^{\mathrm{TM}} 2$, and an Ion PGM ${ }^{\mathrm{TM}}$ sequencer according to the manufacturers' instructions (Life Technologies). One case was examined by using a massively parallel sequencer, the SOLiD system (Life Technologies), as reported previously. ${ }^{4}$

\section{Sanger Sequencing}

DNA samples obtained from frozen tissues or formalin-fixed and paraffin-embedded tissues were used in a Sanger sequencing analysis to validate the RNF43 mutation statuses and detect GNAS and KRAS mutations as described previously. ${ }^{4}$ The primers used to validate the RNF43 mutation statuses are listed in Table 2. The primers used to detect GNAS and KRAS mutations were described previously. ${ }^{4}$

\section{Immunohistochemical Analysis}

Indirect streptavidin-biotin immunohistochemical staining of paraffin-embedded tissues was performed by using a rabbit polyclonal anti-ring finger protein 43 antibody (Atlas Antibodies, Stockholm, Sweden) and a Histofine SAB-PO kit (Nichirei Bioscience Inc., Tokyo, Japan) according to the manufacturers' instructions. To evaluate the staining specificity, a negative control staining condition (no primary antibody) was performed. The immunohistochemical results were evaluated as either reduced or retained expression by comparing the staining intensity of each sample with that of an islet of Langerhans in the same section because the islet of Langerhans showed consistent staining that was easy to identify and evaluate compared with normal ducts that were often devastated by obstructive pancreatitis cause by the neoplasm. The staining was evaluated blindly from sequencing results at lesions with the highest grade of atypia or invasive area in each case. SMA and MAD4 homolog (SMAD4) and tumor protein 53 (TP53) expression were examined by using a mouse monoclonal antiSMAD4 antibody (B-8; Santa Cruz Biotechnology Inc., Santa Cruz, CA, USA) and a mouse monoclonal anti-p53 antibody (DO-7; Dako, Glostrup, Denmark) as described previously. ${ }^{10}$

\section{Statistics}

The statistical analysis incorporated the chi-squared test for comparisons between the molecular and clinicopathological data, and Kaplan-Meier analysis with the log-rank test for survival analyses. Analyses were performed using the PASW Statistics software package (version 18.0; SPSS Inc., Chicago, IL, USA). $P$-values of $<0.05$ were considered as statistically significant.

\section{Results}

We performed sequencing analyses of all coding exons of RNF43 in the 57 patients for whom frozen tissues were available; the analysis was conducted via semiconductor sequencing in 56 samples and via massively parallel sequencing as a whole exome analysis in 1 sample. We obtained the sequence data at an average read depth of 2664 with semiconductor sequencing. The called variations were validated by using tumor DNA, and the somatic state was examined via Sanger sequencing analysis of normal DNA. The massively parallel sequencing data obtained from the single case had been reported previously. ${ }^{4}$ As a result, we found that 8 (14\%) of the 57 intraductal papillary mucinous neoplasms harbored somatic RNF43 mutations, including 5 frameshift mutations, 1 non-sense mutation, and 2 missense mutations (Figure 1; Table 3). Of these mutations, 3 of the 5 frameshift mutations, specifically p.F69fs, p.S264fs, and p.L311fs, as well as the non-sense mutation p.Q153X occurred upstream of 
or within the ring finger domain (residues 272-316 according to the Conserved Domain Database) ${ }^{11}$ of ring finger protein 43 (Figure 1). The remaining two frameshift mutations, p.R363fs and p.V490fs, occurred immediately downstream of the ring finger domain. Of the two missense mutations, p.I164N involved a conserved residue in the proteaseassociated domain (residues 87-186), and p.P310A involved a conserved residue of the ring finger domain, according to the Conserved Domain Database $^{11}$ (Figure 1). SIFT (http://sift.jcvi.org/) ${ }^{12}$ and PolyPhen-2 (http://genetics.bwh.harvard.edu/pph2/ index.shtml), ${ }^{13}$ the online programs used to predict the functional significance of the missense mutations, predicted that both these missense mutations would have damaging effects.

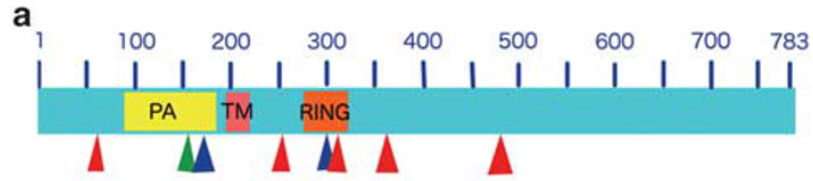

b
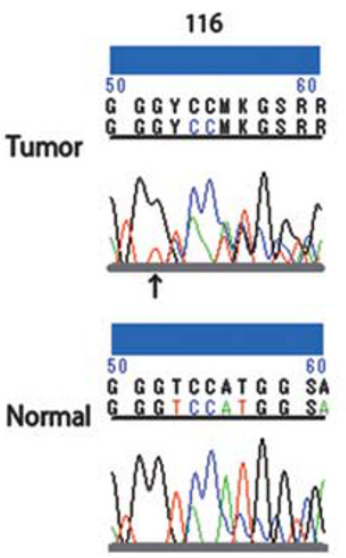

110
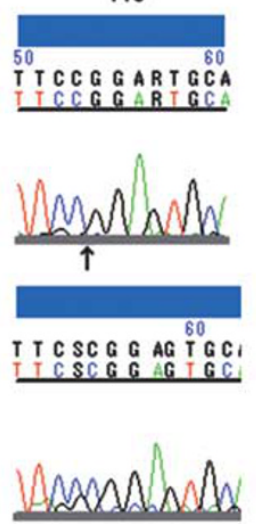

Figure 1 Somatic RNF43 mutations in intraductal papillary mucinous neoplasms. (a) Mutation locations in the ring finger protein 43. Red triangles indicate the frameshift mutations, a green triangle indicates the non-sense mutation, and blue triangles indicate the missense mutations. PA, protease-associated domain; TM, transmembrane domain; and RING, ring finger domain. (b) Representative images of the Sanger sequencing validation. Arrows indicate the c.1087 delC mutation in \#110 and the c.1466delG mutation in \#116.
Next, we investigated the expression of ring finger protein 43 in 176 formalin-fixed and paraffinembedded tissues of intraductal papillary mucinous neoplasms via immunohistochemical analysis. Because intraductal papillary mucinous neoplasm is intrinsically a heterogeneous lesion, some cases showed heterogeneity of staining, so that we evaluated the staining at areas showing the highest grade of atypia or invasive area with comparison with staining of islet cells. As a result, the expression of ring finger protein 43 was reduced in 52 (29.5\%) of the 176 examined tissues relative to the normal islet cells (Figure 2). This reduced expression was significantly associated with the presence of somatic mutations in all but two cases, specifically the case with the p.R363fs frameshift mutation and the case with the p.P310A missense mutation $(P=0.011)$.

We evaluated the association between $R N F 43$ mutations or the expression of ring finger protein 43 and the molecular and clinicopathological features, including GNAS mutations, KRAS mutations, SMAD4 downregulation, TP53 overexpression, tumor grade, histological type, mural nodule detection, macroscopic type, stage, recurrence, and survival. We found that RNF43 mutation was significantly associated with GNAS mutations $(P=0.020)$ and mural nodule detection $(P=0.038$; Table 4$)$. RNF43 expression was not associated with any of the features (Table 4).

\section{Discussion}

In this study, we found that 8 (14\%) of the 57 intraductal papillary mucinous neoplasms harbored somatic RNF43 mutations. Among these mutations, 3 of 5 frameshift mutations and 1 non-sense mutation were expected to yield truncated proteins lacking the protease-associated domain and/or the ring finger domain, whereas 2 missense mutations affected conserved residues in the protease-associated domain or the ring finger domain and would likely result in the functional abrogation of ring finger protein 43. The remaining two frameshift mutations would yield truncated ring finger protein 43 immediately downstream of the ring finger

Table 3 Somatic mutations of RNF43 in intraductal papillary mucinous neoplasms

\begin{tabular}{lllllll}
\hline GRCh37/hg19 & Mutation & Exon & Nucleotide & Protein & Histopathology of IPMN & Type of IPMN \\
\hline ch17:56492734 & Frameshift deletion & Exon 2 & c.204_205delG,T & p.F69CfsX5 & Invasive & Gastric \\
ch17:56440761 & Nonsense & Exon 5 & c.C457T & p.Q153X & Low grade & Gastric \\
ch17:56440727 & Misssense & Exon 5 & c.T491A & p.I164N & High grade & Intestinal \\
ch17:56438207 & Frameshift deletion & Exon 7 & c.786delA & p.S264AfsX155 & Low grade & Gastric \\
ch17:56437534 & Misssense & Exon 8 & c.C928G & p.P310A & High grade & Intestinal \\
ch17:56437530 & Frameshift insertion & Exon 8 & c.931_932insC & p.L311PfsX132 & Invasive & Intestinal \\
ch17:56436050 & Frameshift deletion & Exon 9 & c.1087delC & p.R363GfsX56 & High grade & Intestinal \\
ch17:56435671 & Frameshift deletion & Exon 9 & c.1466delG & p.V490SfsX12 & Low grade & Gastric \\
\hline
\end{tabular}

Abbreviation: IPMN, intraductal papillary mucinous neoplasm. 

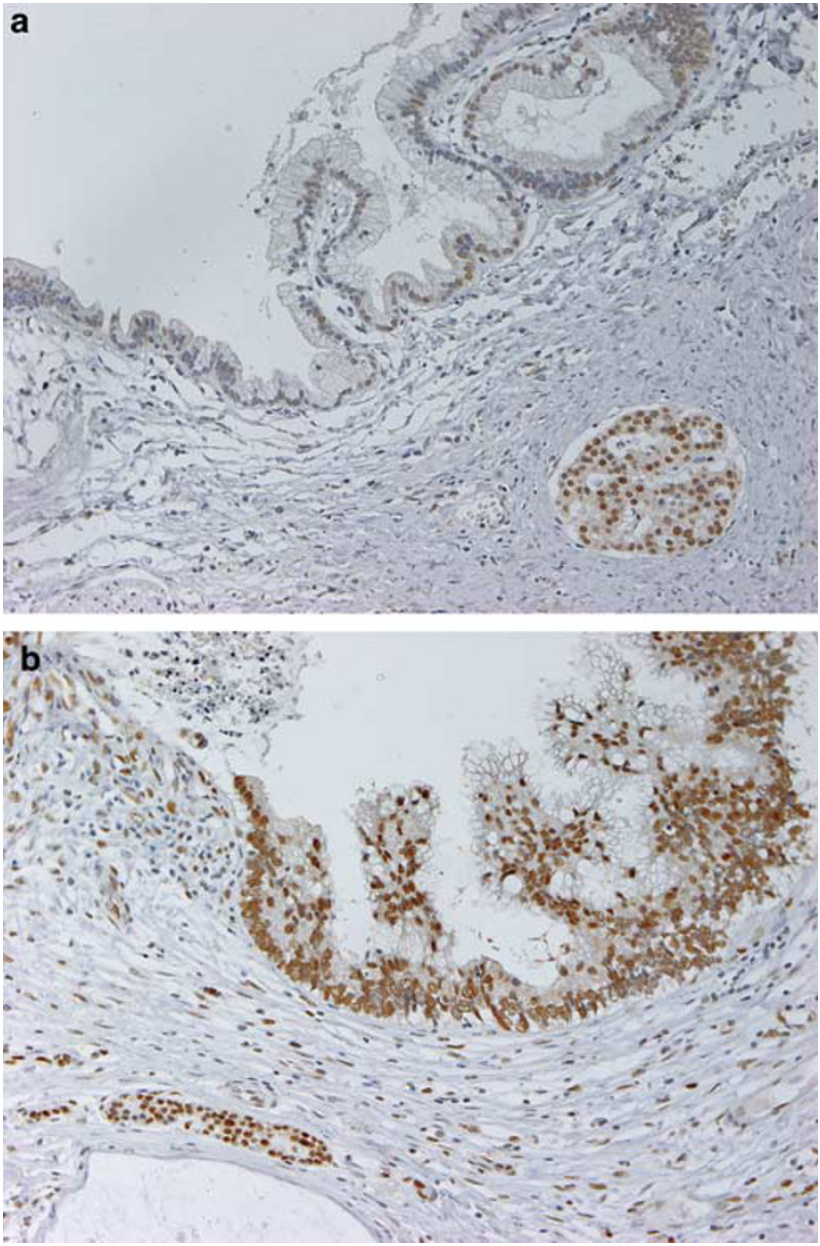

Figure 2 Immunohistochemical analysis indicates the reduced (a) or retained (b) expression of ring finger protein 43 in intraductal papillary mucinous neoplasms. Original magnification, $\times 100$.

domain and would not affect the known functional domains; however, the carboxyl-terminal regions downstream of the ring finger domain were also highly conserved among mammals, according to HomoloGene (http://www.ncbi.nlm.nih.gov/homologene). Moreover, according to the Catalog of Somatic Mutations in Cancer database (http://cancer.sanger. ac.uk/cancergenome/projects/cosmic/), ${ }^{14} 8$ of the 27 reported frameshift mutations in RNF43 involved residues downstream of the ring finger domain, indicating a commonality and suggesting some level of functional significance regarding such mutations. Indeed, Jiang et $a l^{9}$ reported the identification of a frameshift mutation, p.R330fs, that led to the truncation of the encoded protein immediately downstream of the ring finger domain in a pancreatic cancer cell line; this mutation appeared to induce Wnt dependency in the cell, thus indicating the functional abrogation of ring finger protein 43. Nevertheless, most somatic mutations identified in intraductal papillary mucinous neoplasms are loss-of-function mutations, indicat-
Table 4 Associations between RNF43 mutations and clinicopathological features

\begin{tabular}{|c|c|c|c|c|c|c|}
\hline \multirow[b]{2}{*}{ Variable } & \multicolumn{2}{|c|}{$R N F 43$} & \multicolumn{3}{|c|}{ RNF43 expression } & \multirow[b]{2}{*}{ P-value } \\
\hline & Mutant & Wild & P-value & Retain & Reduced & \\
\hline \multicolumn{7}{|l|}{ RNF43 expression } \\
\hline Reduced & 6 & 14 & 0.011 & - & - & \\
\hline Retain & 2 & 35 & & - & - & \\
\hline \multicolumn{7}{|l|}{ GNAS } \\
\hline Mutant & 8 & 28 & 0.020 & 60 & 20 & 0.36 \\
\hline Wild & 0 & 21 & & 66 & 30 & \\
\hline \multicolumn{7}{|l|}{ KRAS } \\
\hline Mutant & 7 & 34 & 0.290 & 66 & 30 & 0.36 \\
\hline Wild & 1 & 15 & & 60 & 20 & \\
\hline \multicolumn{7}{|l|}{ SMAD4 expression } \\
\hline Retain & 7 & 45 & 0.688 & 114 & 40 & 0.058 \\
\hline Loss & 1 & 4 & & 12 & 10 & \\
\hline \multicolumn{7}{|l|}{ TP53 expression } \\
\hline Overexpression & 1 & 12 & 0.454 & 14 & 11 & 0.062 \\
\hline Normal & 7 & 37 & & 112 & 39 & \\
\hline \multicolumn{7}{|l|}{ Histopathology } \\
\hline Low grade & 3 & 27 & 0.448 & 66 & 25 & 0.633 \\
\hline High grade & 3 & 9 & & 28 & 9 & \\
\hline Invasive & 2 & 13 & & 32 & 16 & \\
\hline \multicolumn{7}{|l|}{ Type } \\
\hline Gastric & 4 & 30 & 0.495 & 71 & 30 & 0.133 \\
\hline Intestinal & 4 & 13 & & 45 & 11 & \\
\hline Oncocytic & 0 & 2 & & 4 & 4 & \\
\hline Pancreatobiliary & 0 & 4 & & 6 & 5 & \\
\hline \multicolumn{7}{|l|}{ Mural nodule } \\
\hline Detected & 8 & 31 & 0.038 & 91 & 38 & 0.378 \\
\hline Not detected & 0 & 18 & & 35 & 12 & \\
\hline \multicolumn{7}{|l|}{ Macroscopic type } \\
\hline Branch & 5 & 26 & 0.843 & 62 & 21 & 0.688 \\
\hline Main & 2 & 13 & & 35 & 16 & \\
\hline Mixed & 1 & 10 & & 29 & 13 & \\
\hline \multicolumn{7}{|l|}{ Stage $^{\mathrm{a}}$} \\
\hline $0 \mathrm{a}$ & 3 & 21 & 0.493 & 57 & 24 & 0.553 \\
\hline 0 & 3 & 7 & & 26 & 9 & \\
\hline 1 & 2 & 6 & & 5 & 3 & \\
\hline 2 & 0 & 2 & & 7 & 3 & \\
\hline 3 & 0 & 6 & & 11 & 8 & \\
\hline $4 a$ & 0 & 6 & & 6 & 2 & \\
\hline $4 \mathrm{~b}$ & 0 & 1 & & 3 & 0 & \\
\hline \multicolumn{7}{|l|}{ Recurrence } \\
\hline Recurred & 0 & 8 & 0.218 & 23 & 8 & 0.723 \\
\hline Not recurred & 8 & 41 & & 103 & 42 & \\
\hline \multicolumn{7}{|l|}{ Prognosis } \\
\hline 5-year survival & - & - & & 0.926 & 0.908 & 0.723 \\
\hline
\end{tabular}

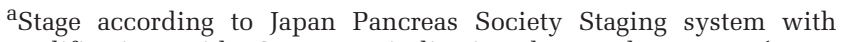
modification with Stage 0a indicating low-grade tumor (Japan Pancreas Society. Classification of Pancreatic Carcinoma. 3rd edn. Kanehara \& Co. Ltd.: Tokyo, 2011).

ing that RNF43 might function as a pancreatic tumor suppressor gene affecting susceptibility linked to intraductal papillary mucinous neoplasm. Recently, Amato et al published a report in which $6(14 \%)$ of 
42 intraductal papillary mucinous neoplasms harbored RNF43 mutations, including 3 frameshift mutations resulting in premature stop codons upstream of the ring finger domain, 2 missense mutations affecting the protease domain and the ring finger domain, and 1 non-sense mutation affecting a residue downstream of the ring finger domain, ${ }^{15}$ thus demonstrating a similar distribution of mutations to that observed in our samples. Wu et al reported that 6 of 8 intraductal papillary mucinous neoplasms harbored RNF43 mutations, all of which were nonsense mutations; 5 of the 6 mutations would generate premature stop codons upstream of the ring finger domain and the remaining mutation, p.R371X, would generate a stop codon after the ring finger domain. ${ }^{5}$ Jiang et $a l^{9}$ identified one non-sense mutation, p.E174X, and one missense mutation, p.F69C, as well as a frameshift mutation, p.R330fs, in pancreatic cancer cell lines, and noted that these mutations appeared to confer Wnt dependency on the cells, thus indicating the functional abrogation of ring finger protein 43 by these mutations. These reports and our current results suggest that loss-offunction RNF43 mutations might play an imperative role in intraductal papillary mucinous neoplasms, possibly by conferring Wnt dependency on the neoplastic cells.

The RNF43 mutations were associated with GNAS mutations and mural nodule detection. GNAS mutations have been found in $40-60 \%$ of intraductal papillary mucinous neoplasms, and are known to be exclusive to these tumors among the various pancreatic neoplasms. ${ }^{4-6}$ Komatsu et $a 1^{16}$ reported that GNAS mutations induced the upregulation of cyclic adenosine monophosphate and overexpression of mucin protein genes in some pancreatic duct-lineage cells; therefore, GNAS mutation appears to play a key role in the secretion of abundant mucin, the most prominent characteristic phenotype of intraductal papillary mucinous neoplasms. Interestingly, the same authors also demonstrated that the exogenous expression of mutated GNAS did not confer a proliferative advantage on pancreatic cancer cells. ${ }^{16}$ These results regarding the association between RNF43 and GNAS mutations might indicate that the loss-of-function of ring finger protein 43 would confer an advantage with respect to proliferation and/or maintenance upon pancreatic ductal cells with GNAS mutations in intraductal papillary mucinous neoplasms. Amato et $a l^{15}$ also indicated that RNF43 mutation was associated with GNAS mutation. The mural nodule indicates the protuberance of a neoplasm into the pancreatic ductal lumen; this is considered to indicate a high-grade lesion. ${ }^{3}$ The association between the mural nodule and RNF43 mutation suggests that ring finger protein 43 might confer proliferative advantages that lead to protruding neoplasms. However, no associations were identified between RNF43 mutation and various clinicopathological features including the histological type, tumor grade, stage, and prognosis, possibly indicating that RNF43 mutation is an early developmental event with no significant impact on the progression of the histological type, dysplastic grade, or tumor stage of intraductal papillary mucinous neoplasms.

We found that $52(29.5 \%)$ of the 176 intraductal papillary mucinous neoplasms exhibited reduced expression of ring finger protein 43 . To the best of our knowledge, this is the first report to reveal aberrant expression of ring finger protein 43 in intraductal papillary mucinous neoplasms. The reduced expression of ring finger protein 43 was associated with RNF43 mutation, which indicates that RNF43 mutation may lead to reduced expression of ring finger protein 43 . Indeed, most RNF43 mutations are truncating mutations and as a result, protein expression will likely be reduced. However, because the reduced expression of ring finger protein 43 was observed more frequently than were mutations, epigenetic alterations might be responsible for this reduced expression in addition to somatic mutations, a possibility that will be addressed in a future study. The reduced protein expression was not associated with any clinicopathological features, indicating that the aberrant expression of ring finger protein 43 might play a role in the development but not in the progression of intraductal papillary mucinous neoplasms.

In conclusion, the results of this study indicate that RNF43 mutation might cause reduced expression of ring finger protein 43 and play a crucial role and associate synergistically with GNAS mutation in development of intraductal papillary mucinous neoplasms of the pancreas.

\section{Acknowledgments}

We thank Yasuaki Akazawa, Mitsuhiro Amemiya, and Akira Saito (StaGen Co. Ltd., Tokyo, Japan) for data processing of next-generation sequencing. This study is supported by the Program for Promoting the Establishment of Strategic Research Centers, Special Coordination Funds for Promoting Science and Technology, Ministry of Education, Culture, Sports, Science and Technology, Japan.

\section{Disclosure/conflict of interest}

The authors declare no conflict of interest.

\section{References}

1 Adsay NV, Fukushima N, Furukawa T, et al. Intraductal neoplasms of the pancreas, In: Bosman FT, Hruban RH, Carneiro F, Theise ND (eds). WHO Classification of Tumours of the Digestive System, 4th edn, Vol., IARC: Lyon; 2010, pp 304-313.

2 Furukawa T, Takahashi T, Kobari M, et al. The mucushypersecreting tumor of the pancreas. Development 
and extension visualized by three-dimensional computerized mapping. Cancer 1992;70:1505-1513.

3 Tanaka M, Fernandez-del Castillo C, Adsay V, et al. International consensus guidelines 2012 for the management of IPMN and MCN of the pancreas. Pancreatology 2012;12:183-197.

4 Furukawa T, Kuboki Y, Tanji E, et al. Whole-exome sequencing uncovers frequent GNAS mutations in intraductal papillary mucinous neoplasms of the pancreas. Sci Rep 2011;1:161.

$5 \mathrm{Wu}$ J, Jiao Y, Dal Molin M, et al. Whole-exome sequencing of neoplastic cysts of the pancreas reveals recurrent mutations in components of ubiquitindependent pathways. Proc Natl Acad Sci USA 2011; 108:21188-21193.

$6 \mathrm{Wu}$ J, Matthaei H, Maitra A, et al. Recurrent GNAS mutations define an unexpected pathway for pancreatic cyst development. Sci Transl Med 2011;3:92ra66.

7 Landis CA, Masters SB, Spada A, et al. GTPase inhibiting mutations activate the alpha chain of Gs and stimulate adenylyl cyclase in human pituitary tumours. Nature 1989;340:692-696.

8 Koo BK, Spit M, Jordens I, et al. Tumour suppressor RNF43 is a stem-cell E3 ligase that induces endocytosis of Wnt receptors. Nature 2012;488:665-669.

9 Jiang X, Hao HX, Growney JD, et al. Inactivating mutations of RNF43 confer Wnt dependency in pancreatic ductal adenocarcinoma. Proc Natl Acad Sci USA 2013;110:12649-12654.
10 Furukawa T, Fujisaki R, Yoshida Y, et al. Distinct progression pathways involving the dysfunction of DUSP6/MKP-3 in pancreatic intraepithelial neoplasia and intraductal papillary-mucinous neoplasms of the pancreas. Mod Pathol 2005;18: 1034-1042.

11 Marchler-Bauer A, Zheng C, Chitsaz F, et al. CDD: conserved domains and protein three-dimensional structure. Nucleic Acids Res 2013;41:D348-D352.

12 Kumar P, Henikoff S, Ng PC. Predicting the effects of coding non-synonymous variants on protein function using the SIFT algorithm. Nat Protoc 2009;4: 1073-1081.

13 Adzhubei IA, Schmidt S, Peshkin L, et al. A method and server for predicting damaging missense mutations. Nat Methods 2010;7:248-249.

14 Forbes SA, Bindal N, Bamford S, et al. COSMIC: mining complete cancer genomes in the Catalogue of Somatic Mutations in Cancer. Nucleic Acids Res 2011;39:D945-D950.

15 Amato E, Molin MD, Mafficini A, et al. Targeted nextgeneration sequencing of cancer genes dissects the molecular profiles of intraductal papillary neoplasms of the pancreas. J Pathol 2014;233:217-227.

16 Komatsu H, Tanji E, Sakata N, et al. A GNAS mutation found in pancreatic intraductal papillary mucinous neoplasms induces drastic alterations of gene expression profiles with upregulation of mucin genes. PLoS ONE 2014;9:e87875. 